University of Nebraska - Lincoln

DigitalCommons@University of Nebraska - Lincoln

8-15-1994

\title{
Dissociative Attachment in Selected Monochloroalkanes
}

D. M. Pearl

University of Nebraska-Lincoln, donpearl363@gmail.com

Paul Burrow

University of Nebraska - Lincoln, pburrow1@unl.edu

Follow this and additional works at: https://digitalcommons.unl.edu/physicsburrow

Part of the Physics Commons

Pearl, D. M. and Burrow, Paul, "Dissociative Attachment in Selected Monochloroalkanes" (1994). Paul Burrow Publications. 12.

https://digitalcommons.unl.edu/physicsburrow/12

This Article is brought to you for free and open access by the Research Papers in Physics and Astronomy at DigitalCommons@University of Nebraska - Lincoln. It has been accepted for inclusion in Paul Burrow Publications by an authorized administrator of DigitalCommons@University of Nebraska - Lincoln. 


\title{
Dissociative attachment in selected monochloroalkanes
}

\author{
D. M. Pearl and P. D. Burrow \\ Department of Physics and Astronomy, University of Nebraska-Lincoln, Lincoln, Nebraska 68588-0111
}

(Received 4 April 1994; accepted 6 May 1994)

\begin{abstract}
The total cross sections for the dissociative attachment (DA) process are reported for eight normal chloroalkanes $\dot{\mathrm{C}}_{n} \mathrm{H}_{2 n+1} \mathrm{Cl}$ for $n=2-9$, two secondary compounds 2-chloropropane and 2-chlorobutane, one tertiary compound $t$-butyl chloride, and three cyclic chloroalkanes $c-\mathrm{C}_{n} \mathrm{H}_{2 n-1} \mathrm{Cl}$ for $n=4-6$, for electron energies below $4 \mathrm{eV}$. Using vertical attachment energies (VAEs) determined by electron transmission spectroscopy elsewhere and in this work, we show that the resonance widths vary linearly with VAE in this series of compounds and that the peak DA cross sections decline exponentially with increasing VAE. The small upper bound to the DA cross section reported previously for methyl chloride is discussed and is consistent with the measurements made on the remaining chloroalkanes.
\end{abstract}

\section{INTRODUCTION}

Measurements of the dissociative attachment (DA) process have been performed on a variety of halogenated hydrocarbon compounds for many years, yet the theory associated with this process in such complex molecules has not been developed. Even in the simplest case of a single halogen atom attached to a hydrocarbon frame, $a b$ initio calculations have not been carried out, although semiempirical methods using diatomic models are making some progress. ${ }^{1}$ To provide motivation for the development of theory and to gain a better understanding of the role played by the hydrocarbon frame, we have made measurements of the DA cross sections of a number of monochloroalkanes, including selections from the normal, secondary, and tertiary alkanes, and the four, five, and six membered ring compounds. Because the impinging electron attaches into the antibonding $\mathrm{C}-\mathrm{Cl} \sigma^{*}$ orbital, these measurements provide a means to investigate the subtle changes that occur in this orbital due to substitution of hydrogen atoms by $\mathrm{CH}_{3}$ and $\mathrm{CH}_{2}$ groups. The more dramatic effects on the DA process due to nearby unsaturated bonds have been touched on earlier by Dressler et al. ${ }^{2}$ and Stricklett et $a .^{3}$ The interaction between the $\mathrm{C}-\mathrm{Cl} \sigma^{*}$ orbital and a more distant $\mathrm{C}=\mathrm{C} \pi^{*}$ orbital has been reported recently by Pearl et al. ${ }^{4}$ using DA as a probe.

Chlorine was chosen as the halogen for this study because the temporary negative ion states associated with oc-. cupation of the $\mathrm{C}-\mathrm{Cl} \sigma^{*}$ orbital, as well as the peaks in the DA cross section, lie well above zero energy. In the heavier halogens, these features appear at lower energies where complications due to unfolding of the finite electron energy distribution may arise. We are not aware of other comprehensive studies having been done previously in the monochloroalkanes, although $\mathrm{CH}_{3} \mathrm{Cl}$ and $\mathrm{C}_{2} \mathrm{H}_{5} \mathrm{Cl}$ have been studied by a number of workers. The attachment processes in a series of normal bromoalkanes have been examined by Christophorou et al. ${ }^{5}$ using a swarm-beam approach. We will comment on this work later in the paper.

\section{EXPERIMENT}

\section{A. Total cross section apparatus}

The present study was performed on two instruments, each of which uses a trochoidal monochromator ${ }^{6}$ to produce a magnetically collimated electron beam. The first, designed for measurement of absolute DA cross sections, is shown schematically in Fig. 1. An electron beam, with currents typically of a few nanoamperes, is injected into the axially symmetric collision region. The ions are produced along the electron path and drift under nearly field-free conditions to the inner collecting cylinder. To minimize the effect of negative ions lost to the endplates, the inner cylinder was made unusually long $(10 \mathrm{~cm})$ with an inner radius of $1 \mathrm{~cm}$. An axial magnetic field of approximately $400 \mathrm{G}$ is applied over the length of the apparatus. Because of the long path length, the alignment of the entrance and exit apertures with the magnetic field is critical to ensure that no part of the electron beam is reflected back into the collision chamber. Misalignment at the exit end creates a larger ion signal and an apparently smaller beam current resulting in an overestimation of the measured cross section. The strength of the magnetic field was chosen to eliminate the collection of elastically scattered electrons by reducing their Larmor radii below the size of the aperture in the exit plate.

If the entrance and exit plates of the collision chamber are biased negatively with respect to the ion collecting cylinder, it is possible for inelastically scattered electrons to become trapped in the potential well and diffuse across the magnetic field lines by subsequent elastic collisions. To eliminate such a "trapped electron" current, the endplates are kept slightly positive with respect to the collision chamber. Care was taken to make sure that this potential did not affect the measured cross sections.

Following the collision chamber, the electron beam is accelerated toward the beam collector which consists of a single plate positioned between two guard electrodes. To minimize the reflection of electrons back into the collision chamber, the collector voltage was increased until the collected beam current was saturated. The reported cross sections were determined to be insensitive to the voltages in the collector region. A potential across two plates parallel to the electron beam could be applied to move reflected electrons or negative ions produced outside the collision chamber off the center axis of the collision chamber and prevent them from reentering the collision chamber, though in practice this was found to be unnecessary.

Except for the collision region, all electrodes exposed to 


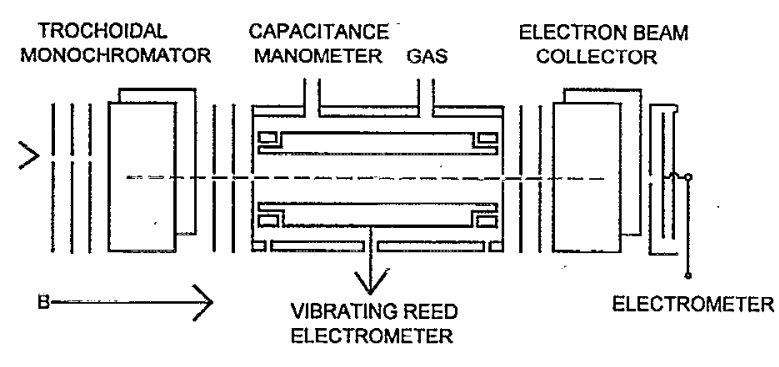

TOTAL DA CROSS SECTION APPARATUS

FIG. 1. A schematic diagram of the total ion collection apparatus with cross sectional view of the collision chamber. The length of the collision chamber is $10 \mathrm{~cm}$.

the electron beam were constructed of molybdenum, which is known to have small potential variations along its surface. The inner cylinder and the entrance and exit electrodes of the collision region were constructed from titanium, which was chosen for its easier machinability compared to molybdenum. It is not known to us whether it is superior in terms of contact potential variations.

Absolute measurement of the pressure inside the collision chamber was made by a capacitance manometer. To minimize thermal transpiration, the apparatus was kept at a temperature near that of the manometer head $\left(45^{\circ} \mathrm{C}\right)$ by means of heating tape wrapped around the outside of the vacuum chamber. The tubing connecting the collision chamber to the manometer head was also heated and kept near this same temperature. All cross sections were measured in a pressure range where the ion current normalized to the electron beam current was linear with pressure. The cross section was measured at a number of different gas pressures in this range with the average of these values reported as the final result.

The performance of the apparatus was verified by measuring the DA cross section of $\mathrm{N}_{2} \mathrm{O}$. The values published in the literature for the $\mathrm{O}^{-}$peak at $2.25 \mathrm{eV}$ are $8.6 \pm 0.6$ and $9.0 \pm 1.4 \times 10^{-19} \mathrm{~cm}^{2}$, by Rapp and Briglia ${ }^{7}$ and Krishnakumar and Srivastava, ${ }^{8}$ respectively. Our result of $8.5 \pm 0.7 \times 10^{-19} \mathrm{~cm}^{2}$ is within $2 \%$ of the cross section reported by Rapp and Briglia. ${ }^{7}$ Because of the extreme sensitivity of the apparatus to alignment, this measurement was performed periodically to guarantee proper operation.

The standard deviation of the measured cross sections for each compound was no more than $7 \%$ of the average value. The móst significant systematic error is likely to be the loss of ions taking place at the ends of the collecting cylinder. ${ }^{9}$ For our geometry, we estimate that the fraction of ions lost should be no more than $7 \%$, assuming they are isotropically generated. Because our test cross section in $\mathrm{N}_{2} \mathrm{O}$ lies so close to that of Rapp and Briglia, ${ }^{7}$ however, we are reluctant to blindly apply this correction without further study. Other sources of systematic error come from measurement of the beam current, gas pressure and temperature, each of which amounts to $1 \%-2 \%$. Combining the secondary sources of systematic error with the random measurement error, we believe the reported cross sections to be accurate within $10 \%$.

The same apparatus is used in a second mode of operation which measures the derivative with respect to electron energy of the current transmitted through the collision chamber, a technique first devised by Sanche and Schulz ${ }^{10}$ for locating the sharp structures in the total scattering cross section due to the formation of temporary negative ion states. In electron transmission spectroscopy (ETS), a small alternating voltage is applied to the inner cylinder to modulate the impact energy of the electron beam, and the a.c. component of the transmitted electron current, which is proportional to the derivative of the transmitted current, is detected with a lock-in amplifier. In the present study, ETS measurements were carried out in a number of the chlornalkanes to locate the energies of the resonances associated with temporary occupation of the $\mathrm{C}-\mathrm{Cl} \sigma^{*}$ orbitals.

\section{B. Ion counting apparatus}

The second apparatus, which has been déscribed previously, ${ }^{11,12}$ contains an effusive molecular beam crošsed with a magnetically collimated electron beam. Ions are pushed from the collision region by a potential applied to a semicylindrical electrode on one side of the electron beam and pass through an electrostatic lens system into a chevron package of two microchannel plates. A cylindrical set of fine shield wires surround the electron beam to reduce the potential drop across the electron beam due to the pusher voltage.

A portion of the ion current observed in this apparatus arises from electron collisions with the background gas. In particular, a sharp peak near zero energy was observed in all of the compounds, which is apparently produced, in part, from a reaction of the compound with the hot tungsten filament that is used as the electron source. To eliminate this background signal, a branch in the gas inlet system was created such that the sample could be introduced either into the collision region or directly into the background. The difference between these two sets of measurements is assumed to be free of impurities generated in the background.

The second apparatus has several operational advantages over the absolute measurement system previously described. The resolution of the electron beam is significantly, better due to the lower magnetic fields needed for operation and the shorter overall length of the scattering region. Furthermore, it is possible to observe the DA cross sections at electron energies near zero energy without interference from elastically scattered or trapped electrons. We have, therefore, used this apparatus to determine the shapes of the relative cross sections for DA and normalized the results to the peak cross sections as measured on the first apparatus. The energy calibration of the DA cross section is also conveniently accomplished in this apparatus by reference to the sharp peak near zero energy: The convolution of a Gaussian distribution with a full width at half-maximum (FWHM) of $67 \mathrm{meV}$, used to approximate the electron energy distribution, with a rapidly rising cross section for electron energies approaching zero yields a peak in the cross section at $20 \mathrm{meV}^{13}$ and the data presented here have been shifted accordingly. We estimate that the energy scale is accurate to within $\pm 50 \mathrm{meV}$. This 


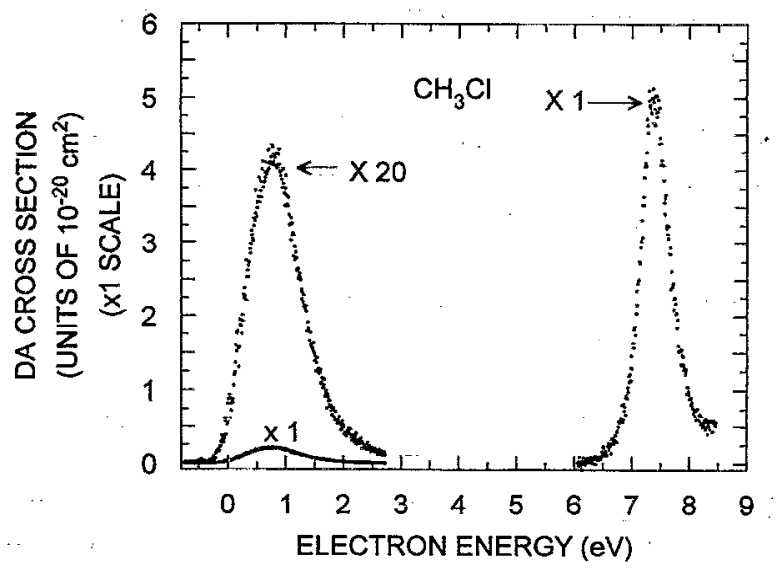

FIG. 2. The apparent DA cross section of methyl chloride as a function of electron impact energy.

method of energy calibration was chosen as there is no convenient sharp feature in the DA cross sections of common gases to use as a reference, and it is unlikely that the peak energies are known to better than $\pm 50 \mathrm{meV}$ in any case. Also, many DA peaks, such as that for $\mathrm{O}^{-}$from $\mathrm{N}_{2} \mathrm{O}$, lie at energies which overlap with the peak in the monochloroalkanes.

Except for methyl and ethyl chloride, all of the compounds in this study are liquids at room temperature. The latter were subjected to three freeze-thaw cycles to eliminate

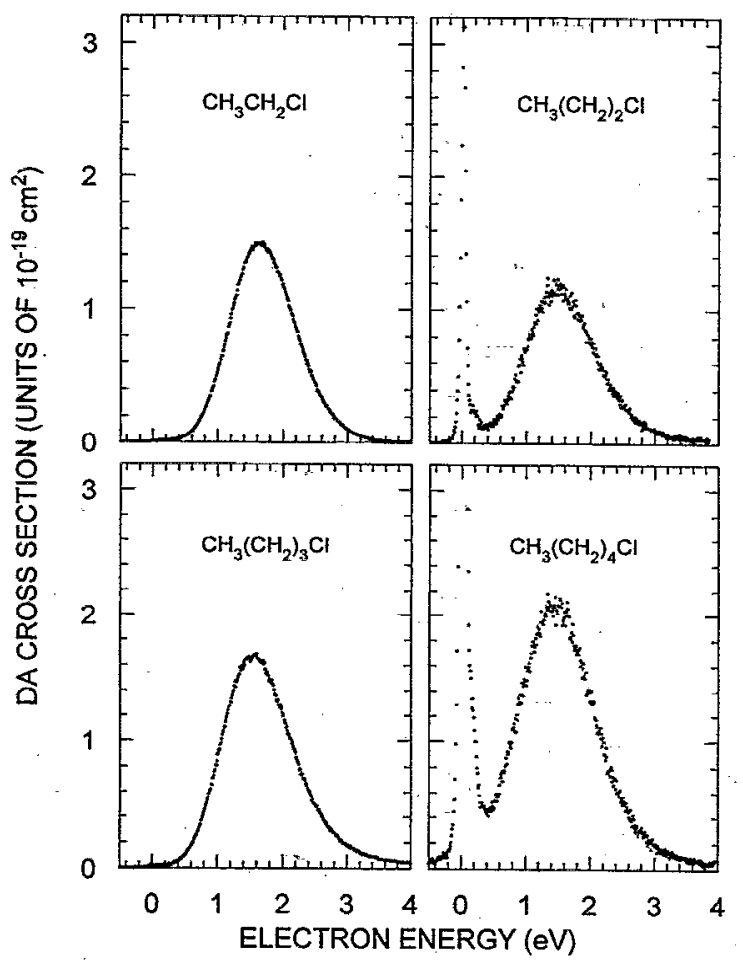

FIG. 3. The DA cross section as a function of electron impact energy for $\mathrm{C}_{n} \mathrm{H}_{2 n+1} \mathrm{Cl}$ with $n=2-5$. The peak near zero energy in this figure and Figs. $4-6$ is due to an impurity.

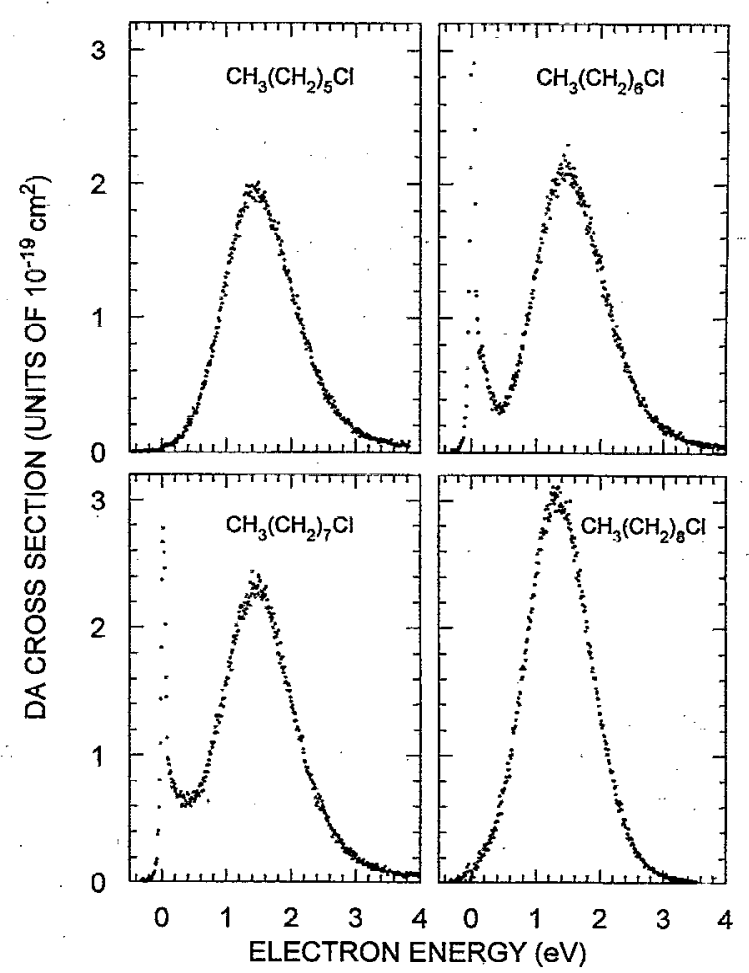

FIG. 4. The DA cross section as a function of electron impact energy for $\mathrm{C}_{n} \mathrm{H}_{2 n+1} \mathrm{Cl}$ with $n=6-9$.

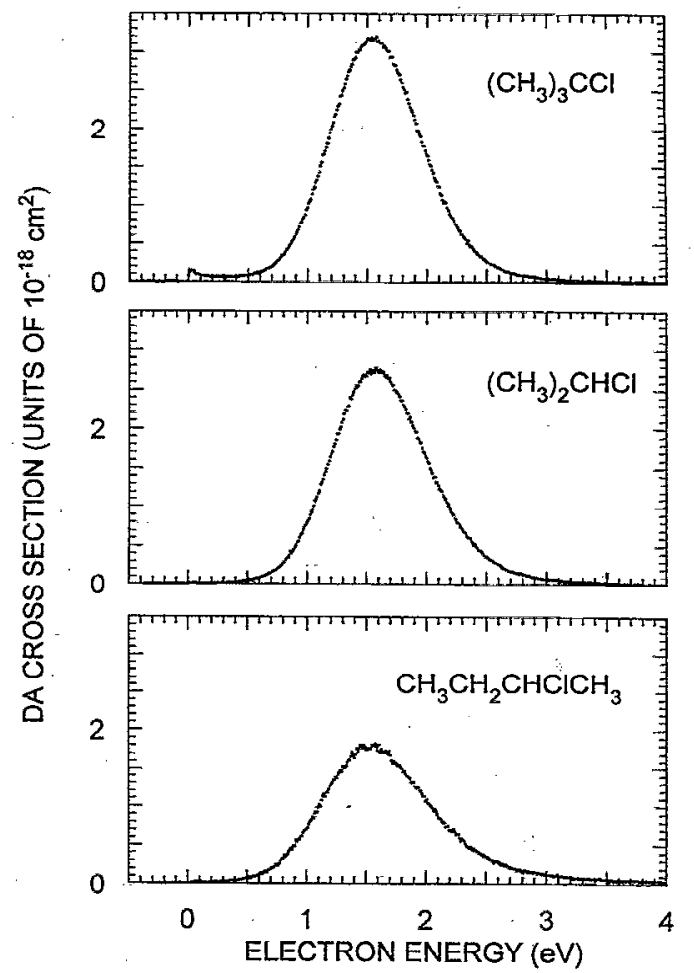

FIG. 5. The DA cross section as a function of electron impact energy for $t$-butyl chloride, 2-chloropropane, and 2-chlorobutane. 


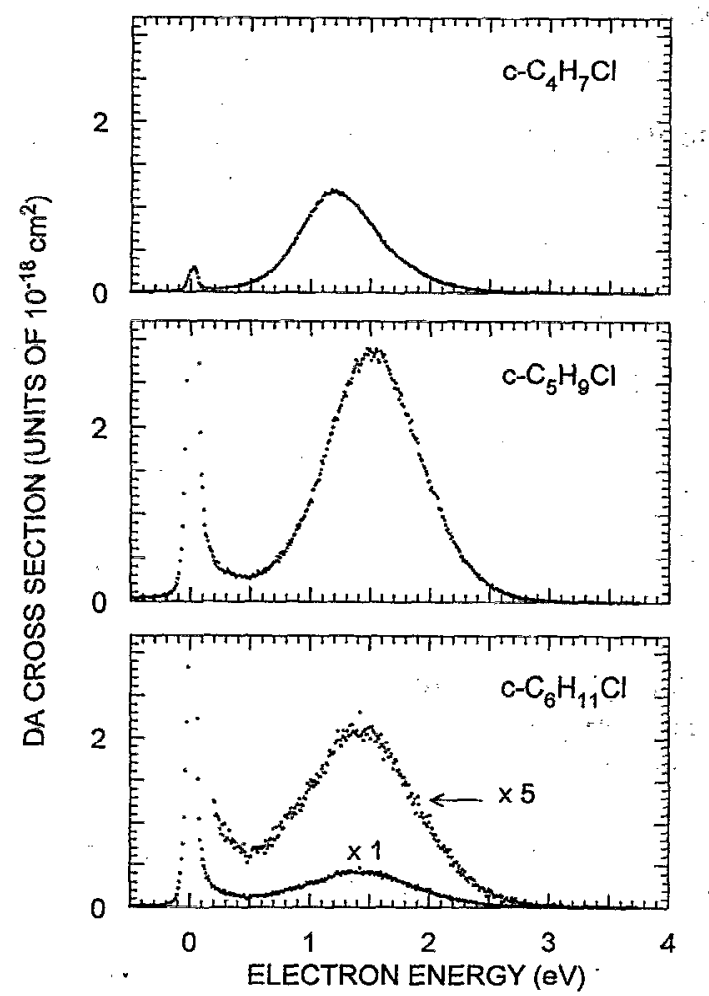

FIG. 6. The DA cross section as a function of electron impact energy for $c-\mathrm{C}_{n} \mathrm{H}_{2 n-1} \mathrm{Cl}$ with $n=4-6$. In the lower panel, the cross section scale applies to the $\times 1$ curve.

trapped gases: In a number of the compounds, the anion current showed a sharp peak near zero energy even after subtracting the background contribution. Though the purity of these compounds; as given by Aldrich Chemical Company, is typically $99 \%$, we believe that the source of this peak is a contaminant in the sample. For several of the compounds, allowing the sample to evaporate slowly into the vacuum system for several hours eventually eliminated this peak. In the others, the peak size would significantly decrease after several hours of evaporation, but was never completely eliminated. To verify that the zero energy peak was due to an impurity, a sample of 1-chlorononane was purified chromatographically ${ }^{14}$ and the peak was indeed removed from the DA cross section. It seems likely that the impurity is $\mathrm{CCl}_{4}$ which has a very large cross section peaking at zero energy. 11

Finally, we assume that the anion fragments produced in $\mathrm{DA}$ are all $\mathrm{Cl}^{-}$at the low impact energies employed here. We have not verified this with a mass analyzer, but it seems unlikely that a significant amount of other charged fragments would be created since large electron affinities would be required. In a DA study of methyl chloride using a mass analyzer, Scheunemann et al. ${ }^{15}$ saw only $\mathrm{Cl}^{-}$produced below $6 \mathrm{eV}$.

\section{RESULTS}

In this section, we present the results of our DA measurements. The dependence of the DA cross section on inci-
TABLE I. Peak dissociative attachment cross sections and peak cnergies; and vertical attachment energies and resonance widths.

\begin{tabular}{|c|c|c|c|c|}
\hline Compound & $\begin{array}{c}\text { DA peak } \\
\text { cross section } \\
\left(10^{-19} \mathrm{~cm}^{2}\right)\end{array}$ & $\begin{array}{c}\text { DA peak } \\
\text { energy } \\
(\mathrm{eV})\end{array}$ & $\begin{array}{c}\text { VAE } \\
(\mathrm{ETS}) \\
(\mathrm{eV})\end{array}$ & $\begin{array}{c}\text { ETS FWHM } \\
(\mathrm{eV})\end{array}$ \\
\hline Methyl chloride & $<0.02$ & 0.8 & $3.45,^{b} 3.3^{c}$ & $2.3,^{b} 2.7^{c}$ \\
\hline Ethyl chloride & 1.49 & 1.55 & $2.35^{\mathrm{a}}$ & $1.8^{\mathrm{a}}$ \\
\hline 1-Chloropropane & 1.17 & 1.50 & 2.4 & $1.8^{\mathrm{a}}$ \\
\hline 1-Chlorobutane - & 1.65 & 1.54 & $2.39^{\mathrm{a}}$ & $1.8^{\mathrm{a}}$ \\
\hline I-Chloropentane & 2.08 & $1: 47 \cdots$ & $2.26^{\mathrm{a}}$ & $1.78^{\mathrm{a}}$ \\
\hline 1-Chlorohexane & 1.99 & 1.43 & $=$ & \\
\hline 1-Chloroheptane & 2.13 & 1.47 & & \\
\hline 1-Chlorooctane & 2.30 & $1.45^{\circ}$ & $2.24^{\mathrm{e}}$ & $1.76^{\circ}$ \\
\hline 1-Chlorononane & 3.01 & 1.34 & $2.23^{\mathrm{e}}$ & $1.75^{\mathrm{e}}$ \\
\hline 2-Chloropropane & 27.6 . & 1.58 & $1.99^{\mathrm{a}}$ & $1.35^{\mathrm{a}}$ \\
\hline 2 Chlorobutane & 17.8 & 1.51 & $2.05^{\mathrm{a}}$ & $1.4^{\mathrm{a}}$ \\
\hline$t$-Butyl chloride & 31.8 & 1.55 & $1.80^{\mathrm{d}}$ & $1.20^{\mathrm{d}}$ \\
\hline Cyclobutyl chloride & 11.8 & 1.19 & $1,98^{\mathrm{e}}$ & $1.4^{c}$ \\
\hline Cyclopentyl chloride & 28.3 & 1.52 & $1.93^{\circ}$ & $1.35^{\mathrm{e}}$ \\
\hline Cyclohexyl chloride & 4.13 & 1.43 & $2.31^{\circ}$ & $1.70^{\circ}$ \\
\hline
\end{tabular}

${ }^{a}$ Guerra et al. (Ref, 16). "Modelli et al. (Ref. 18).

${ }^{b}$ Burrow et al. (Ref. 17) (ETS). $\quad$ Present work.

'Shi et al. (Ref. 23) (VIB).

dent electron energy is shown for methyl chloride, from our previous work, ${ }^{2}$ in Fig. 2, the normal alkanes in Figs. 3 and 4 , the secondary and tertiary compounds in Fig. 5, and the ring compounds in Fig. 6. In all cases, the sharp peak at zero energy is believed to be spurious. With the exception of methyl chloride, these compounds all display DA peaks which are very similar in shape. The peak magnitudes and energies are listed in Table I:

The energies and a measure of the lifetimes of the temporary negative ion states that undergo dissociation are conveniently determined by ETS. The energies of the peaks in the total scattering cross sections yield the vertical attachment energies (VAEs) for formation of the temporary negative ion states. The widths of these peaks are inversely related to the resonance' lifetimes and are determined from the difference in energy between the minimum and maximum in the derivative of the transmitted current as the electron energy is swept through the resonance. ${ }^{10}$ The VAEs and widths are also listed in Table $\mathrm{I}$. The values for $\mathrm{CH}_{3}\left(\mathrm{CH}_{2}\right)_{n} \mathrm{Cl}(n=1-3),\left(\mathrm{CH}_{3}\right)_{2} \mathrm{CHCl}$, and $\mathrm{CH}_{3} \mathrm{CH}_{2} \mathrm{CHClCH}_{3}$ were determined by Guerra et al. ${ }^{16}$ for $\mathrm{CH}_{3} \mathrm{Cl}$ by Burrow et al.,${ }^{17}$ for $\left(\mathrm{CH}_{3}\right)_{3} \mathrm{CCl}$ by Modelli et al. ${ }^{18}$ and the remainder were measured in the total collection apparatus described earlier using the ETS mode of operation.

Briefly, the trends in the data are as follows: First, in the normal alkanes, the peak cross sections increase with the length of the hydrocarbon frame, although not monotonically. Second, the peak cross sections for the two secondary chloroalkanes are larger by a factor of roughly 10 than the average of the normal chloroalkanes. Third, the tertiary hydrocarbon $t$-butyl chloride has a peak cross section 15 times larger than the average of the normal chloroalkanes. Last, the cyclic compounds span a range of DA cross sections, with 
that of cyclohexyl chloride closer to those of the normal chloroalkanes and cyclobutyl and cyclopentyl chloride similar to the secondary and tertiary compounds.

We note that these results are in good general agreement with those of Guerra et al. ${ }^{16}$ who, in addition to measuring the VAEs of a number of the chloroalkanes using ETS, made relative observations of the DA cross sections in several of them. The production of $\mathrm{Cl}^{-}$from $t$-butyl chloride, 2-chloropropane, and the normal alkyl chlorides was in the approximate proportions 15,10 , and 1 , respectively, in agreement with our absolute measurements. Furthermore; Guerra et al. ${ }^{16}$ observed and noted the shifts in VAEs and resonance widths that take place upon substitution of $\mathrm{a}_{3} \mathrm{CH}_{3}$ or larger alkyl group for a $\mathrm{H}$ atom.

Measurement of the DA cross section of methyl chloride, the simplest of all the chloroalkanes, has proven to be very difficult and warrants a brief review. Several investigators have reported a large ${ }^{15}$ or small ${ }^{11}$ peak at zero energy. We have shown previously, ${ }^{12}$ however, that this peak vanishes with elimination of $\mathrm{CCl}_{4}$ from the system and careful accounting of the signal arising from the background. As shown in Fig. 2, there remains a small peak at $0.8 \mathrm{eV}$. Although this peak is too small to observe in our total collection apparatus, we have measured the DA cross section of a higher-lying peak at $7.4 \mathrm{eV}$. Assuming the kinetic energy distributions of the $\mathrm{Cl}^{-}$fragments are not too dissimilar for these two processes, the ratio of the peaks permits us to make a rough estimate of $2 \times 10^{-21} \mathrm{~cm}^{2}$ for the $0.8 \mathrm{eV}$ feature. Nevertheless, we remain unconvinced that this cross section is due to pure methyl chloride since semiempirical calculations by Fabrikant ${ }^{1}$ yield a cross section at room temperature that is about three orders of magnitude smaller.

\section{DISCUSSION}

The molecular properties that influence DA cross sections of diatomics are well known ${ }^{19-21}$ and can be extended to larger polyatomics. The most significant of these are the energy and lifetime of the temporary anion state. Both properties are complicated functions of the electronic structure of the molecule, the symmetry and shape of the temporarily occupied orbital, and the molecular polarizability and dipole moment. Other considerations for a series of related compounds are the relative bond strengths and lengths, reduced masses of the fragments, conformations, and other steric factors. The DA cross section can be most simply expressed as ${ }^{20}$

$$
\sigma_{\mathrm{DA}}=\sigma_{\mathrm{CAP}} S \text {, }
$$

where $\sigma_{\mathrm{DA}}$ is the DA cross section, $\sigma_{\mathrm{CAP}}$ is the capture cross section for formation of the temporary negative ion, and $S$ is the survival factor giving the probability that the ion will live long enough to dissociate. In a diatomic model, the survival factor can be expressed as ${ }^{19,20}$

$$
S=\exp \left[-\int_{R_{e}}^{R_{c}} \frac{\Gamma_{a}(R)}{\hbar} \frac{d R}{v(R)}\right],
$$

where $R$ is the distance between the two atoms, $R_{e}$ is their separation when the electron first attaches, and $R_{c}$ is the separation at which the neutral and anion curves cross. $v(R)$ is the velocity with which the atoms separate and $\Gamma_{a}$ is the total autodetachment width. By approximating the positiondependent width with an average value, a cruder but more illuminating expression for the survival factor may be given

$$
S \approx \exp \left(-\frac{\tau_{s}}{\bar{\tau}_{a}}\right)
$$

where the separation time is:

$$
\tau_{s}=\int_{R_{e}}^{R_{c}} \frac{d R}{v(R)},
$$

and the average lifetime of the anion state is

$$
\bar{\tau}_{a}=\frac{\hbar}{\bar{\Gamma}_{a}}
$$

Although the DA cross section depends on many different characteristics of the molecule, it is likely that differences in the survival factor will be of most importance in the chloroalkanes studied in the present work. For small DA cross sections, such as those measured here, the survival factor itself is small and exponentially dependent on the resonance lifetime.

The lifetime of the anion state is determined by the length of time necessary for the captured electron to tunnel out of the potential that binds it to the molecule. The effective potential is formed by the combined effect of the polarizability, dipole moment, and the centripetal barrier due to the angular momentum components of the electron. ${ }^{22}$ To the extent that the shape of the $\mathrm{C}-\mathrm{Cl} \sigma^{*}$ molecular orbital is much the same in all these compounds, we expect that the centripetal barrier will be similar as well. The lifetime of the anion state, therefore, will depend primarily on the energy of the temporary anion and its location with respect to this barrier.

The relationship between the anion energies and lifetimes may be illustrated using the VAEs and resonance widths as measured using ETS. Although a portion of the width is due to the Franck-Condon overlap between the ground state nuclear wave function and that of the repulsive potential curve of the anion, we assume that this does not vary substantially among the monochloroalkanes. In Fig. 7, we plot the widths of the resonances vs the VAEs, where the different symbols designate the type of compound. For very broad resonances, such as that in $\mathrm{CH}_{3} \mathrm{Cl}$, the width determined from ETS is subject to error because the signal derives from the total scattering cross section and variations in the nonresonant part of the scattering may shift the energies of the extrema in the derivative of the transmitted current. For this reason, we include the width of the $\mathrm{CH}_{3} \mathrm{Cl}$ anion state as it appears in the vibrational excitation function of the $\mathrm{C}-\mathrm{Cl}$ stretch mode as measured by Shi et al. ${ }^{23}$ In this channel, there is no interference with contributions from nonresonant scattering. As observed in Fig. 7, this more reliable value, indicated by (VIB), lies higher than that determined by ETS. The energy of the peak is also shifted to somewhat lower energy. Overall, the data seem to be surprisingly well fit by a linear relationship between the resonance width and VAE with a slope of 1.0. An extension of the line in Fig. 7 to 


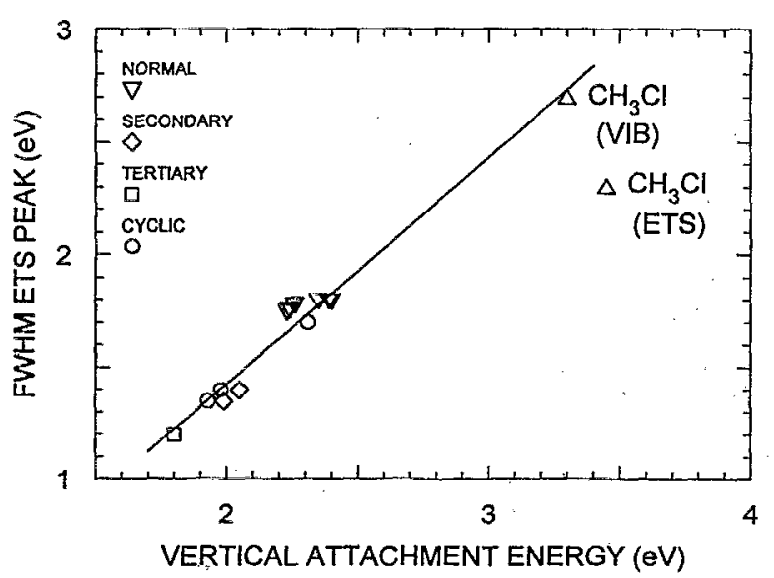

FIG. 7. The full width at half-maximum of the resonance peaks as a function of vertical attachment energy as measured using electron transmission spectroscopy. A more reliable value for $\mathrm{CH}_{3} \mathrm{Cl}$ is also given, labeled (VIB), determined from the vibrational excitation cross section measured by Shi et al. (Ref. 23).

lower values of $\mathrm{VAE}$, however, indicates that near $\mathrm{VAE}=0.5$ $\mathrm{eV}$, the predicted resonance width is zero. This result is unphysical and suggests that linearity is not maintained for VAE $<1.5 \mathrm{eV}$.

Figure 7 implies that the anion lifetime is inversely proportional to the VAE. If the separation times were the same for all the molecules, this would imply that the survival probabilities, and hence the DA cross sections, vary exponentially with VAE. We illustrate this in Fig. 8, which shows the log of the peak DA cross sections plotted against the VAEs for all of the compounds in this study for which we have values. The line drawn in Fig. 8 is a best fit to the data excluding methyl chloride, for which we have only an upper limit to the true cross section. Figure 8 provides compelling evidence that the primary molecular property determining the size of the DA peak in the monochloroalkanes is the VAE of the anion state. However, the considerable scatter in the data,

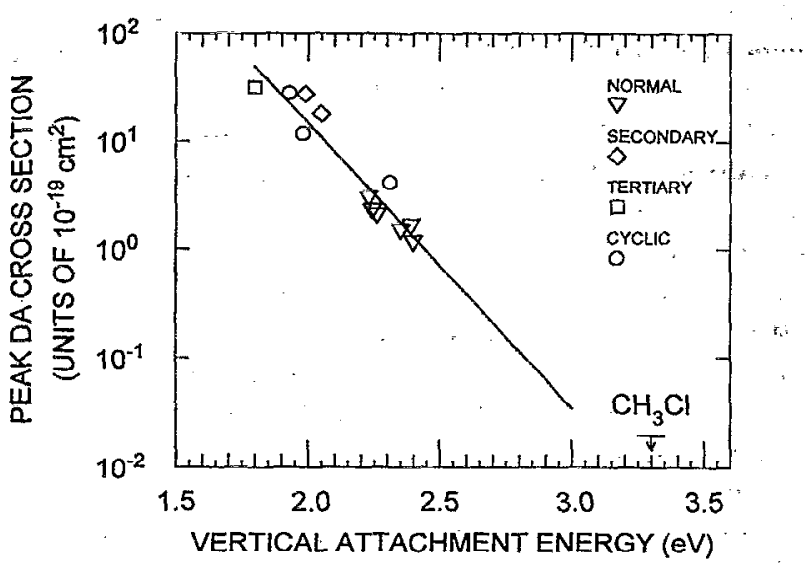

FIG. 8. A semilog plot of the peak DA cross section as a function of the VAE. The line represents a least squares fit to the data, not including $\mathrm{CH}_{3} \mathrm{Cl}$. The DA peak cross section given for $\mathrm{CH}_{3} \mathrm{Cl}$ is an experimental upper limit only.

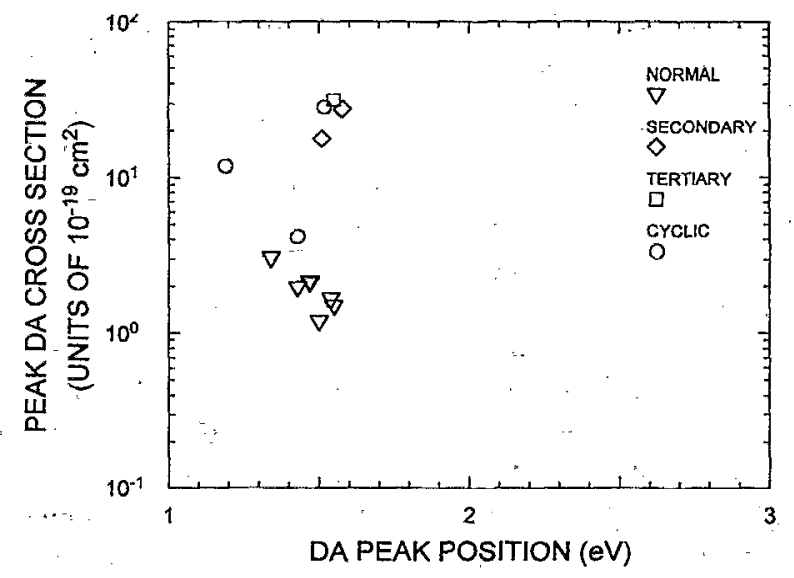

FIG. 9. A semilog plot of the peak DA cross section as a function of DA peak energy.

disguised in part by the semilog plot, indicates that other factors are clearly at work. The average deviation from the line for all the compounds is approximately $40 \%$, well outside our experimental error.

In early attempts to correlate DA cross sections with temporary negative. ion properties over a broad range of molecules, ${ }^{24}$ the cnergies of the peaks in the DA cross sections were used, rather than the VAEs, which were largely unknown. This treatment neglects the shifts in energy caused by the survival factor in compounds with short anion lifetime, and the correlation becomes much less obvious. This is illustrated in Fig. 9 in which the $\log$ of the maximum DA cross section is plotted against the energies of the peaks in the $D A$ cross section. The spread in peak energies is much smaller, with most of the data clustered $\sim 1.5 \mathrm{eV}$.

In addition to the dependence of the survival factor on the VAE, the separation time $\tau_{s}$ also appears in the exponential, as noted in Eq. (3). This factor depends in a complex manner on the shapes of the neutral and anion potential surfaces and the reduced mass of the two fragments. For diatomic molecules, the anion potential may be approximated by a linear region connecting the energy at the equilibrium separation to that at the crossing between the neutral and anion curve. This permits a simple expression for $\tau_{s}$ to be derived in terms of these energies, the change in internuclear separation, and the reduced mass. Thus far, however, we have not found a way of scaling our data, using this simplification, to correct for differences in reduced mass that improves the scatter appearing in Fig. 8. The four butyl compounds, e.g., which have the same reduced mass, do not fall appreciably closer to a single line in Fig. 8 than the other compounds. Although this may be a conisequence of our crude estimates of the potentials and neglect of other factors, it may also be that the diatomic approximation is not valid.

\section{A. Selected normal, secondary, and tertiary compounds}

Figure 8 clearly indicates that the molecular properties determining the energy of the temporary negative ion state will most profoundly affect the maximum DA cross section. In the present work, this is most evident in the series methyl, 
ethyl, 2-propyl, and $t$-butyl chloride. In these compounds, the carbon atom attached to the chlorine changes its bonding partners successively from three hydrogen atoms to three methyl groups. The progressive lowering of the anion state energy associated with the $\mathrm{C}-\mathrm{Cl} \sigma^{*}$ orbital arises from two effects. First, the strength of the $\mathrm{C}-\mathrm{Cl}$ bond decreases slightly ${ }^{25,26}$ and the bond length increases, ${ }^{27}$ shifting the potential curve of the neutral molecule, again pictured as a quasidiatomic, closer to the repulsive potential curve of the anion. Second, replacement of a $\mathrm{C}-\mathrm{H}$ bond with a more polarizable and lower lying $\mathrm{C}-\mathrm{C}$ bond should cause a greater perturbation of the $\mathrm{C}-\mathrm{Cl} \sigma^{*}$ orbital, forcing it downward in energy. Although energies computed ${ }^{28}$ using Koopmans' theorem and the Slater-type orbital (STO)-3G basis set order the $\mathrm{C}-\mathrm{C} \sigma^{*}$ orbitals below the $\mathrm{C}-\mathrm{H} \sigma^{*}$ orbitals, they do not indicate a shift in $\mathrm{C}-\mathrm{Cl} \sigma^{*}$ orbital energy in going from methyl to ethyl chloride. This seems surprising to us since Falcetta and $\operatorname{Jordan}^{29}$ have shown that the STO-3G basis set yields results for the relative ordering of the $\mathrm{C}-\mathrm{Cl} \sigma^{*}$ anion states of the chloromethanes that are in reasonably good agreement with those of more sophisticated approaches using larger basis sets and stabilization techniques. On the other hand, the multiple scattering $X_{\alpha}$ calculations employed by the Italian group ${ }^{16,18}$ show that the $\mathrm{C}-\mathrm{Cl} \sigma^{*}$ orbital in $t$-butyl chloride is stabilized with respect to that in methyl chloride, although the mechanism for this shift was not discussed.

As might be anticipated, substitution of a $\mathrm{C}-\mathrm{H} \sigma^{*}$ orbital by a $\mathrm{C}-\mathrm{C} \sigma^{*}$ orbital has its greatest effect in going from methyl to ethyl chloride, producing a downward shift of 1.1 $\mathrm{eV}$ in the VAE and a DA cross section change of several orders of magnitude. From ethyl to 2-chloropropane, the VAE drops by $0.36 \mathrm{eV}$ and the peak cross section increases by a factor of 19. Finally, $t$-butyl chloride has the lowest VAE, $0.19 \mathrm{eV}$ lower than that of 2-chloropropane, and the largest peak cross section.

We note that the trends in the DA cross section are consistent with measurements of the reductive cleavage of carbon-halogen bonds examined by cyclic voltammetry. ${ }^{30}$ These liquid phase studies report that cleavage is easiest in the order tertiary $>$ secondary $>$ normal.

\section{B. The normal chloroalkanes}

Although attachment rate constant measurements in ethyl chloride have been made at low energies, ${ }^{31}$ we know of no electron beam studies at higher energies in the normal chloroalkanes with which to compare our absolute cross sections. The peak DA cross sections of the normal chloroalkanes from ethyl chloride to 1-chlorononane are plotted in Fig. 10 as a function of the reduced mass of the chlorine atom and the remaining radical. The trend is clearly upward with increasing chain length, which is counter to the strongest mass effect in the DA process. ${ }^{19,20}$ In diatomic molecules with small survival factors, an increase in the reduced mass creates an increased separation time and consequently a decreasing trend for the DA cross section as in the case of the series $\mathrm{H}_{2}, \mathrm{HD}$, and $\mathrm{D}_{2}{ }^{32}$

A second mass effect may arise because of factors in the capture cross section which vary as $M_{r}^{1 / 4} E^{-1}$, where $M_{r}$ is the reduced mass and $E$ is the energy of the impinging

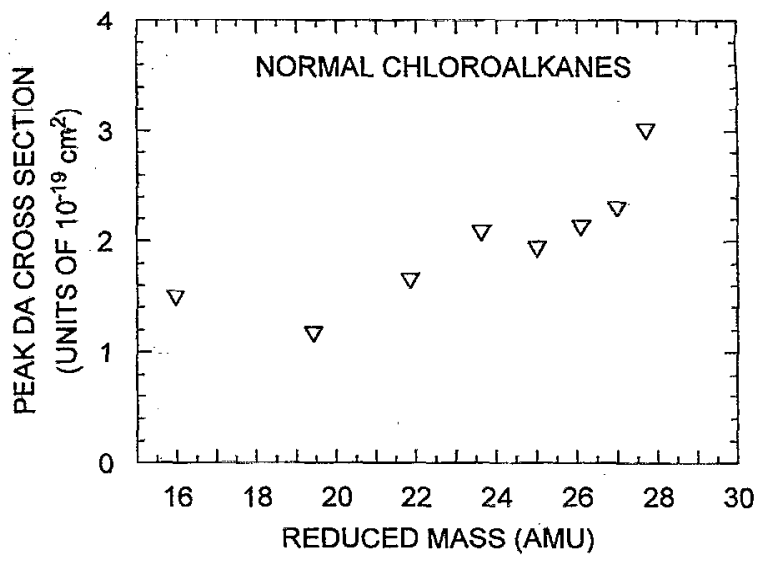

FIG. 10. A plot of the peak DA cross sections as a function of reduced mass for $\mathrm{C}_{n} \mathrm{H}_{2 n-1} \mathrm{Cl}$ with $n=2-9$.

electron. ${ }^{19,20}$ This small effect is normally observable only when the survival factor is near unity; otherwise; it is swamped by the effect of the reduced mass on the separation time. Because the peak DA cross sections in the monochloroalkanes are small, and hence the survival factor is much less than unity, these compounds are not expected to display such an effect. This was further tested by plotting a "reduced" cross section scaled by dividing out the dependence on $M_{r}^{1 / 4} E^{-1}$. The resulting plot still showed a trend toward higher values with increasing mass.

The considerations above, as well as the variation of the cross section with VAE seen in Fig. 8, suggest to us that as the number of $\mathrm{C}-\mathrm{C}$ bonds increases, additional $\mathrm{C}-\mathrm{C} \sigma^{*}$ orbitals are introduced which depress the $\mathrm{C}-\mathrm{Cl} \sigma^{*}$ orbital energy and thereby increase the survival factor. The polarizability of the alkyl chain increases with length and will also act to depress the VAE. One would expect these effects to gradually saturate, however, there are also an increasing.number of molecular conformations in these compounds which must be considered.

Using a swarm-beam method, Christophorou et al..$^{5}$ have measured the DA cross sections of a series of normal bromoalkanes with two to six and eight carbon atoms. The cross sections for the two to six compounds are approximately 30 times larger than those in the corresponding chloroalkanes, but display a similar increasing trend with larger reduced masses. Christophorou et al..$^{5}$ suggest that these results are not inconsistent with the $M_{r}^{1 / 4} E^{-1}$ behavior discussed earlier. However, this was based on assumptions yielding an erroneously large value of the temporary negative ion lifetime. Because of this, they concluded that the survival factor was near unity and that the reduced mass dependence of the DA cross section would be determined by factors in the capture cross sections.

Only the VAEs for ethyl bromide, 1-bromopropane, and $t$-butyl bromide have been reported. ${ }^{18,33}$ These lie near 1.2 $\mathrm{eV}$, well above the energies of the peaks at $0.73 \mathrm{eV}$ in the DA cross sections reported by Christophorou et al. ${ }^{5}$ The rather substantial differences in energy imply that the survival factor is well below unity. It is interesting to note that the VAEs 
for ethyl bromide and $t$-butyl bromide differ only by $0.14 \mathrm{eV}$, a much narrower range than the $0.6 \mathrm{eV}$ difference found in the corresponding chlorine bearing compounds. This is consistent with our earlier picture in that the $\mathrm{C}-\mathrm{Br} \sigma^{*}$ orbital lies much lower than the $\mathrm{C}-\mathrm{Cl} \sigma^{*}$ orbital and thus is less sensitive to perturbations brought about by the introduction of the $\mathrm{C}-\mathrm{C} \sigma^{*}$ orbitals.

The 1-bromooctane cross section determined by Christophorou et al..$^{5}$ appears to be quite anomalous. Its magnitude is about five times larger than that of the remaining bromoalkanes, and the peak is shifted substantially lower in energy, from $\sim 0.73 \mathrm{eV}$, where the two to six compounds are clustered to $0.13 \mathrm{eV}$. This compound deserves further scrutiny to determine whether the measurement was affected by an impurity, since, from our measurements in the chloro compounds, a major shift in the DA peak energy would be quite unexpected. On the other hand, if the result can be verified, it might provide evidence for an unusual conformation.

\section{The cyclic chloroalkanes}

The DA cross sections of cyclobutyl and cyclopentyl chloride fall in the range anticipated for the secondary compounds, along with 2-chloropropane and 2-chlorobutane. Cyclohexyl chloride, on the other hand, has a VAE and peak DA cross section closer to those of the normal chloroalkanes. Compared to the four and five membered rings, cyclohexyl chloride is unstrained and the $\mathrm{C}-\mathrm{C}$.bond angles are close to that found between the carbon atoms in the normal compounds. However, 2-chloropropane and 2-chlorobutane are also unstrained and thus it seems unlikely that this effect alone accounts for the difference in behavior. Molecular orbital calculations exploring ring compounds and their VAEs would be useful.

\section{v. CONCLUSIONS}

In the present work, we have shown more explicitly the close connection between dissociative attachment cross sections and vertical attachment energies and resonance widths determined by electron transmission spectroscopy in the monochloroalkanes. In particular, we observe a linear relationship between inverse lifetimes and VAE, and an exponential decline of the peak DA cross section with increasing VAE. Our data illustrate how the $\mathrm{C}-\mathrm{Cl} \sigma^{*}$ orbital can be "tuned" to lower energies by substitution on the primary carbon, thus increasing the DA cross section. Stabilization of the anion state by other means, such as placement of the molecules on a metal surface or in a polarizable medium, should also greatly enhance the DA cross sections, particularly in compounds such as methyl chloride that have such small cross sections in the unperturbed state.

Although our results are consistent with a small DA cross section in methyl chloride, the actual value of the cross section at room temperature is still uncertain. The line drawn in Fig. 8 , if projected to the VAE determined in the vibrational excitation cross section, yields a cross section of $5.7 \times 10^{-22} \mathrm{~cm}^{2}$. This value is $70 \%$ lower than our experi- mental upper limit placed on this peak, but more than two orders of magnitude greater than that calculated by Fabrikant. $^{1}$

The dispersion of the data points in Figs. 7 and 8 reveals that there are several effects not accounted for in our discussions, e.g., steric effects that might hinder access to the $\mathrm{C}-\mathrm{Cl}$ $\sigma^{*}$ orbital by the impinging electron and variations in the capture cross sections. The DA process in polyatomic molecules continues to offer theorists a considerable challenge in several areas, such as $a b$ initio calculations of the resonance energies and lifetimes, the anion potential surface near the crossing with that of the neutral molecule, and the handling of multiple vibrational modes.

\section{ACKNOWLEDGMENTS}

This work was supported by the National Science foundation through grants CHE-9300941 and CHE-8922601. We are pleased to acknowledge many useful conversations and advice from Gordon Gallup, Ilya Fabrikant, Kenneth Jordan, and John Nash.

${ }^{1}$ I. I. Fabrikant, J. Phys. B 24, 2213 (1991).

${ }^{2}$ R. Dressler, M. Allan, and E. Haselbach, Chimia 39, 285 (1985).

${ }^{3}$ K. L. Stricklett, S. C. Chu, and P. D. Burrow, Chem. Phys. Lett. 131, 279 (1986).

${ }^{4}$ D. M. Pearl, P. D. Burrow, J. J. Nash, H. Morrison, and K. D. Jordan, J. Am. Chem. Soc. 115, 9876 (1993).

${ }^{5}$ L. G. Christophorou, J. G. Carter, P. M. Collins, and A. A. Christodoulides, J. Chem. Phys. 54, 4706 (1970).

${ }^{6}$ A. Stamatovic and G. J. Schulz, Rev. Sci. Instrum. 41, 423 (1970).

${ }^{7}$ D. Rapp and D. D. Briglia, J. Chem. Phys. 43, 1480 (1965).

${ }^{8}$ E. Krishnakumar and S. K. Srivastava, Phys. Rev. A 41, 2445 (1990).

${ }^{9}$ H.-X. Wan. J. H. Moore, and J. A. Tossell, J. Chem. Phys. 94, 1868' (1991).

${ }^{10}$ L. Sanche and G. J. Schulz, Phys. Rev. A 5, 1672 (1972).

${ }^{11}$ S. C. Chu and P. D. Burrow, Chem. Phys. Lett. 172, 17 (1990).

${ }^{12}$ D. M. Pearl and P. D. Burrow, Chem. Phys. Lett. 206, 483 (1993).

${ }^{13}$ I. I. Fabrikant (private communication).

${ }^{14}$ We are indebted to J. J. Nash at Purdue University for purifying 1-chlorononane for us.

${ }^{15} \mathrm{H}$.-U. Scheunemann; E. Illenberger, and H. Baumgaertel, Ber. Bunsenges. Phys. Chem. 84, 580 (1980); S. K. Srivastava and O. J. Orient, in Third International Symposium on Production and Neutralization of Negative Ions and Beams, edited by Krsto Prelec (American Institute of Physics, Brookhaven, NY, 1983), p. 56.

${ }^{16}$ M. Guerra, D. Jones, G. Distefano, F. Scagnolari, and A. Modelli, J. Chem. Phys. 94, 484 (1991)

${ }^{17}$ P. D. Burrow, A. Modelli, N. S. Chiu, and K. D. Jordan, J. Chem. Phys. 77, 2699 (1982).

${ }^{18}$ A. Modelli, F. Scagnolari, G. Distefano, M. Guerra, and D. Jones, Chem. Phys. 145, 89 (1990).

${ }^{19}$ T. F. O'Malley, Phys. Rev. 150, 150 (1966).

${ }^{20}$ J. N. Bardsley, A. Herzenberg, and F. Mandl, Proc. Phys. Soc. London 89, 321 (1966).

${ }^{21}$ J. C. Y. Chen, Phys. Rev. 148, 66 (1966).

${ }^{22}$ F. H. Read, J. Phys. B (Proc. Phys. Soc.) 2, 893 (1968).

${ }^{23}$ X. Shi, V. K. Chan, and P. D. Burrow (unpublished).

${ }^{24}$ L. G. Christophorou and J. A. D. Stockdale, J. Chem. Phys. 48, 1956 (1968).

${ }^{25}$ K. W. Egger and A. T. Cocks, Helv. Chim. Acta 56, 1516 (1973).

${ }^{26} \mathrm{~J}$. D. Cox and G. Pilcher, Thermochemistry of Organic and Organometallic Compounds (Academic, New York, 1970).

${ }^{27}$ R. H. Schwendeman, G. D. Jacobs, and J. M. Kraigs, J. Chem. Phys. 40, 1022 (1964).

${ }^{28} \mathrm{G}$. Gallup (private communication). 
${ }^{29}$ M. F. Falcetta and K. D. Jordan, J. Phys. Chem. 94, 5666 (1990).

${ }^{30}$ C. P. Andrieux, I. Gallardo, J.-M. Savéant, and K.-B. Su, J. Am. Chem. Soc. 108, 638 (1986).

${ }^{31} \mathrm{Z}$. Lj. Petrovic, W. C. Wang, and L. C. Lee, J. Chem. Phys. 90, 3145 (1989). In ethyl chloride; these authors report a peak in the cross section of $1.5 \times 10^{-19} \mathrm{~cm}^{2}$ at $0.12 \mathrm{eV}$ which we do not observe.

${ }^{32}$ G. J. Schulz and R. K. Asundi, Phys. Rev. Lett. 15, 946 (1965); Phys. Rev. 158, 25 (1967)

${ }^{33}$ A. Modelli, F. Scagnolari, G. Distefano, D. Jones, and M. Guerra, J. Chem. Phys. 96, 2061 (1992). 